\title{
Optical Techniques that can be Applied to Investigate GDI Engine Combustion
}

\author{
Richard Stone, Ben Williams and Paul Ewart
}

University of Oxford

\begin{abstract}
The increased efficiency and specific output with Gasoline Direct Injection (GDI) engines are well known, but so too are the higher levels of Particulate Matter emissions compared with Port Fuel Injection (PFI) engines. To minimise Particulate Matter emissions, then it is necessary to understand and control the mixture preparation process, and important insights into GDI engine mixture preparation and combustion can be obtained from optical access engines. Such data is also crucial for validating models that predict flows, sprays and air fuel ratio distributions. The purpose of this paper is to review a number of optical techniques; the interpretation of the results is engine specific so will not be covered here. Mie scattering can be used for semi-quantitative measurements of the fuel spray and this can be followed with Planar Laser Induced Fluorescence (PLIF) for determining the air fuel ratio and temperature distributions. With PLIF, very careful in-situ calibration is needed, and for temperature this can be provided by Laser Induced Thermal Grating Spectroscopy (LITGS). LITGS temperature measurements can also be used to quantify the differences in evaporative cooling with different fuels, thereby explaining observed changes in the volumetric efficiency. Natural light photography can be used for tracking flame fronts, and with suitable calibration of the colour filter array in a high speed colour video camera, then soot pyrometry can be employed to estimate the temperature and soot loading. The rich information derived from optical techniques drives their proliferation in research.
\end{abstract}

\section{Introduction}

The Bowditch system [1] for optical access (Figure 1) is widely used and enables an increasingly broad range of diagnostic techniques that are available, and this is coupled with the increasing performance of high speed digital cameras. The optical techniques can be divided into laser based techniques and those that use high speed photography (perhaps with lasers as an illumination source). The techniques to be described here are ones that have been used at Oxford, and in some cases originated at Oxford. The emphasis will be on the techniques and not the results, as the results that are included here are merely for illustration of the techniques. The discussion will start with high speed photography (both with natural light and external illumination), and then discuss laser based techniques for point and 2D measurements. Some of these techniques (and indeed many others) are described by Zhao [2], but others are more recent or outside the scope of this book. The engine used in the work reported here is based on the Jaguar AJ133 Gasoline Direct Injection (GDI) engine [3] with the specification given in Table 1

Table 1 Specification of the optical access engine

\begin{tabular}{ll}
\hline Type & 4-stroke \\
Combustion system & Direct Injection \\
Bore x Stroke & $89 \times 90 \mathrm{~mm}$ \\
Swept volume & $562 \mathrm{~cm} 3$ \\
Compression & ratio $11: 1$ \\
Fuel pressure & $150 \mathrm{bar}$
\end{tabular}

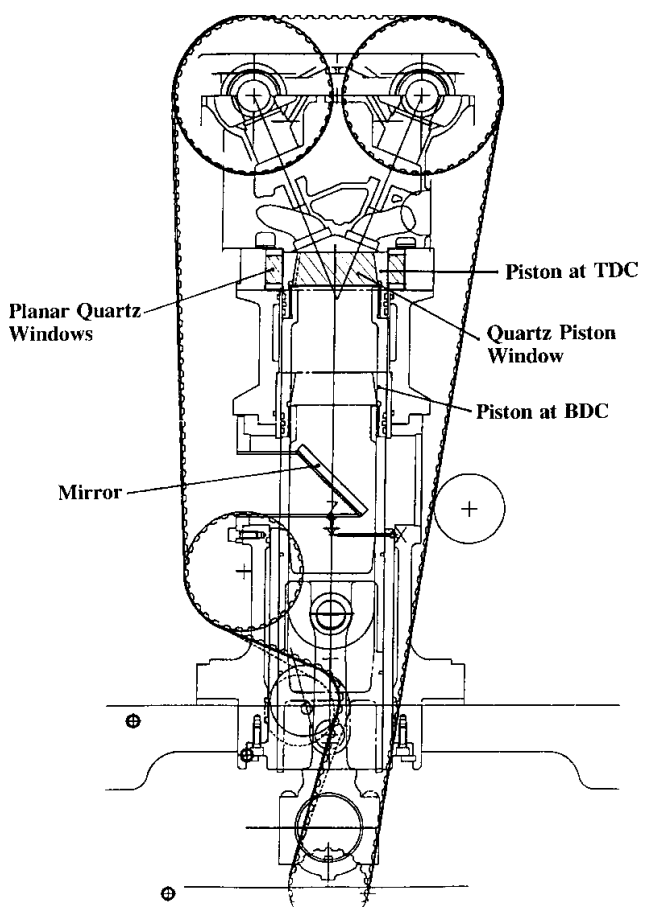

Figure 1 Bowditch arrangement to allow optical access through the piston crown.

The discussion of optical techniques starts with what might be called natural light techniques combined with high speed photography. This will cover spray imaging, full-bore flame front imaging and soot pyrometry. The section on laser based techniques is restricted to point temperature measurements and Planar Laser Induced Fluorescence (PLIF). It is explained how PLIF can be used for quantitative measurements of air fuel ratio, and then how this can be used for temperature measurements. The huge challenge with PLIF is in achieving a robust calibration 


\section{Natural light techniques}

\section{Fuel spray imaging}

Fuel spray imaging can either be by back illumination (shadowgraphy, in which the light source is behind a screen to diffuse the light) or by direct light scattering from the droplets (Mie scattering). For a single droplet the intensity of scattered light is proportional to the surface area, but in a spray there will be multiple scattering (and obscuration) and the lighting intensity will not be uniform, so quantitative measurements are challenging to envisage. Furthermore, both types of result also depend on the illumination source, the camera settings and the nature of any image postprocessing. With a consistent approach to the imaging system and the image post-processing, then the results can become semi-quantitative and be useful for side-by-side comparisons of different injectors, fuels, and temperatures and pressures of the fuel and air. The steps in image processing can include:

1 Obtaining a reference image before fuel injection for background subtraction

2 In a sample image, identify the injector location and the approximate positions of the sprays. This is so that a region outside the spray can be monitored for changes in background illumination (due to changes in light source or scattered light - this is especially important with Mie scattering), and the spray region can be interrogated for 'connectivity' so as to identify the fuel spray

3 Apply image processing to a sequence of images so as to obtain spray angles and tip penetration, and apply false colouring to the fuel spray, this requires

a. applying a threshold to binarize the image

b. using a connectivity algorithm to identify the fuel spray, and apply false colouring to the fuel spray

c. superimpose the coloured fuel spray onto a greyscale image that shows features such as the injector location and valves.

Some further details are in [4] along with examples of quantitative measurements.

Figure 2 shows a side-by-side comparison of Mie scattering and shadowgraphy, along with the use of false colouring of the spray. Although the human eye can distinguish minute differences in a greyscale, visualisation is aided by using about 10 distinct bands and when colour is used the visual interpretation and impact are enhanced. The light source here was a $100 \mathrm{~W}$ LED array overdriven by a factor of about 6 (since the duration was less than $10 \mathrm{~ms}$ ), enabling very short exposures (of order $10 \mu \mathrm{s}$ ) and a small aperture so as to give a good depth of field. When imaging combustion in the same cycle a much lower intensity illumination is needed since the exposure might be $200 \mu$ s with an aperture of $f / 2$, as the chemiluminescence from a flame is orders of magnitude less intense.

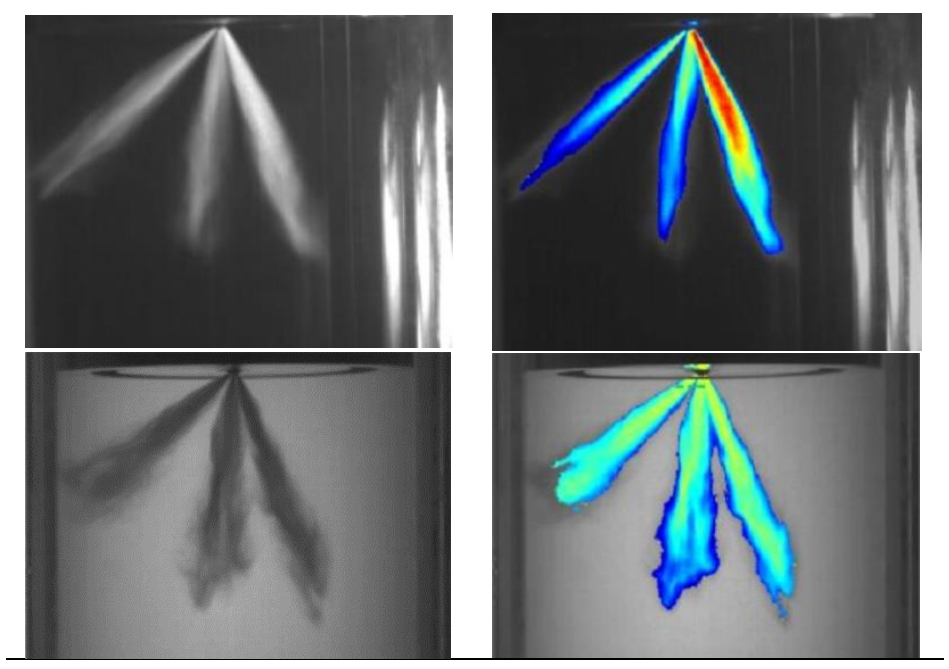

Figure 2 Mie scattering (upper) and shadowgraphy images of a fuel spray for the same conditions, with false colouring applied to the fuel spray

When a sequence of images is obtained then the 'quantity' of fuel present in the spray can be tracked by summing the greyscale values of each pixel within the fuel spray. This allows comparisons between the same fuel but with different coolant or air temperatures (or indeed between different fuels). Figure 3 makes a comparison between different splash blends of ethanol, and since multiple images have been taken, then the mean values have been plotted, along with bars to indicate the standard deviation. 
When the fuel is made to fluoresce, then the intensity of the fluorescence can be proportional to the volume of liquid fuel. If this is combined with Mie scattering, then this leads to techniques that use simultaneous measurements to estimate the droplet size. The ratio of the signal from the two techniques is theoretically proportional to the local Sauter Mean Diameter, provided multiple scatterings are not significant, and this has been referred to as Laser Sheet Dropsizing [6].

\section{Combustion imaging}

The two sources of light from flames are chemiluminescence (blue and UV) and thermal radiation from soot. As the thermal radiation is in the IR and the red end of the visible light spectrum, then a colour camera can distinguish between these two different sources. That said, the camera provides a line-of-sight integration, and the thermal radiation is much more intense than chemiluminescence, so the thermal radiation can obscure the chemiluminescence even when a homogeneous mixture is only slightly rich of stoichiometric.

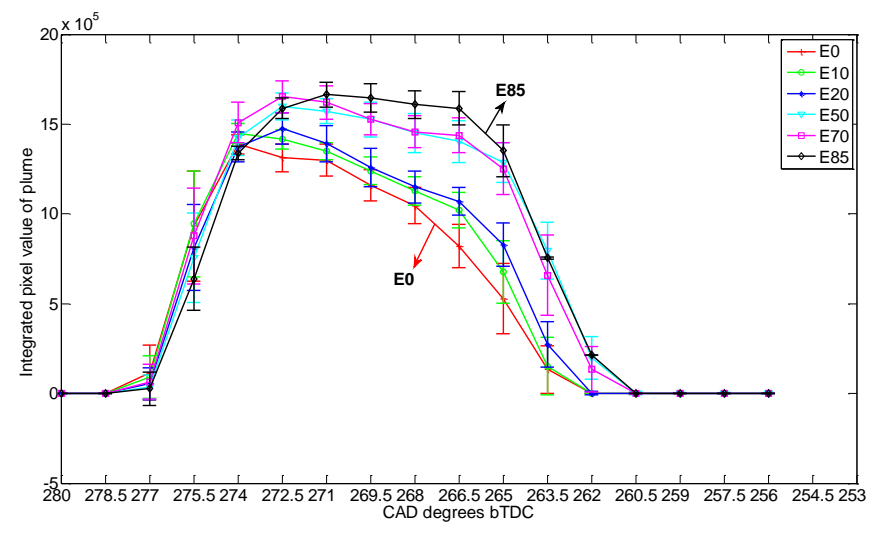

Figure 3 Integrated pixel values as a function of crank angle for gasoline/ ethanol blends with $80^{\circ} \mathrm{C}$ coolant (E20 means $20 \%$ ethanol by volume); the bars associated with the average values are \pm 1 standard deviation [5]

A conventional Bowditch piston has an aperture of about $70 \%$ of the bore diameter (or $50 \%$ of the bore area), but this does not allow much of the flame growth to be tracked, primarily because of the density ratio across the flame front, meaning that only the first few percent of the mass fraction to be burned can be tracked. This is none the less useful because the pressure based analysis of the early stages of combustion are very sensitive to errors and assumptions in the analysis. Such imaging is also useful when looking at the interaction of fuel sprays and ignition when studying split injection that is being used to develop a stratified charge, such as in catalyst light-off strategies [7]. Another technique that is useful (but not strictly imaging) is the use of interference filters with photomultiplier tubes to detect chemiluminescence from species such as $\mathrm{C} 2$ (515 $\mathrm{nm}$ ) and $\mathrm{CH}$ $(430 \mathrm{~nm})$ to establish whether a misfire has occurred because of a failure of the flame to propagate (as opposed to failure to ignite) [7]. Closely related to this is the detection of chemiluminescence from $\mathrm{CN}$ (at $388 \mathrm{~nm}$ ), as this can be normalized by the spark-discharge energy during the observation interval (typically $150 \mu \mathrm{s}$ at the start of the glow discharge), so as to estimate the air fuel ratio in the arc between the spark plug electrodes [8].

The limitations of the standard Bowditch piston window can be overcome by using a window with a concave lower surface [9] as shown in Fig 4. This of course leads to optical distortion of the image that will be a function of the piston position. However, with digital systems the piston position will be known for each image and distortion correction can be applied to the individual images that are then reassembled into a video.

The procedure for full-bore imaging is:

1 photograph a reference target without the piston in place

2 photograph the reference target through the piston at a sequence of crank angles

3 Determine the correction for each crank angle transformation by using control point selection and local weighted mean (LWM) spatial transformation. Cubic interpolation rather than linear was used on the coordinates of the selected control points at known crank angles to derive the transformations for other crank positions within $0-120^{\circ} \mathrm{CA}$. Crop the image to remove 'ghost images' outside the field of view and reassemble the images into a video.

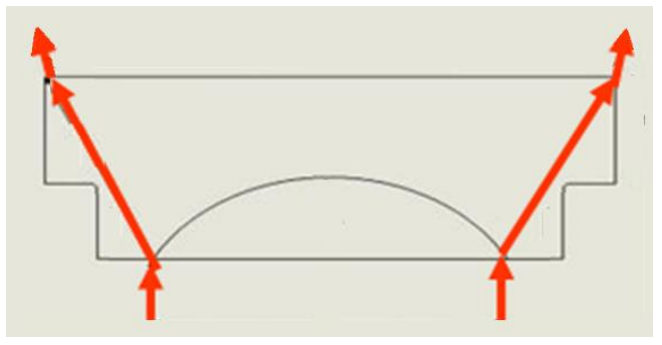

Figure 4 Use of a concave lower surface on the piston window so as to give full-bore imaging4 Use of a concave lower surface on the piston window so as to give full-bore imaging

Algorithms can then be used to identify the flame front location, the enflamed area and the locus of the centroid of the area. This is not as straightforward as it might appear since there is noise present in the images and the flame front needs to be continuous. To improve the signal to noise ratio in the flame image sequences and avoid picking up redundant or spurious flame edges, the images were smoothed using nonlinear diffusion filtering before applying an edge detecting algorithm. Non-linear diffusion filtering is a very powerful image processing tool for image noise reduction and image simplification. The method is based on the original approach established by Perona and Malik [10]. The principle is to smooth out noise locally by diffusive flow while at the same time preventing flow across object boundaries (edges). Occasionally, the edge detection routine generated 'holes' within the detected boundary or 'spots' outside it. These were due to extremely bright pixel clusters such as a cloud of soot. Median filtering and region filling were then applied in MATLAB to remove these false features and make the boundary a simple closed contour. The median filtering process also helped to smooth the edge and eliminate redundant shape features. Some sample results are shown in Figure 5. 

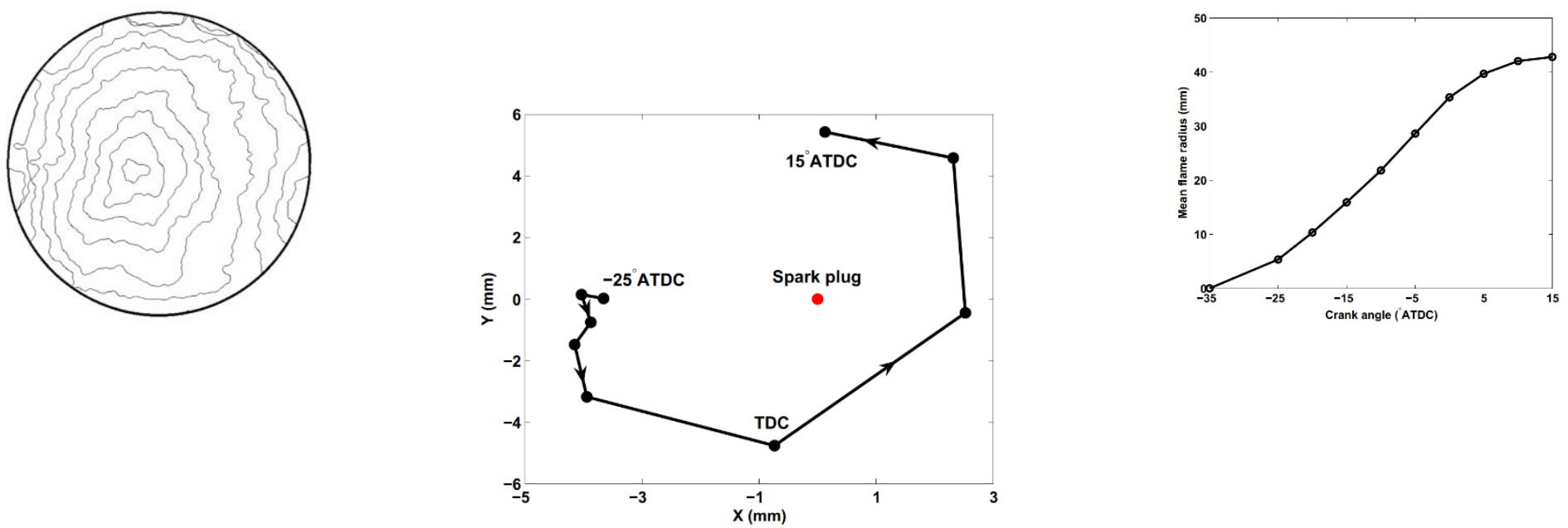

Figure 5 Flame fronts from $25^{\circ} \mathrm{BTDC}$ to $15^{\circ} \mathrm{ATDC}$ in steps of $5^{\circ} \mathrm{CA}$, with toluene and $\lambda=1.2$ (top), and analysis of flame growth with mean flame radius (middle) and flame centroid locus (bottom)

\section{Three Colour Pyrometry}

Soot pyrometry relies on the relationship between the temperature of a glowing particle and the intensity of the thermal radiation it emits. The spectral intensity emitted by a black body, $I_{b}$, is a function of wavelength and temperature alone, and is given by Planck's Radiation law:

$(1$

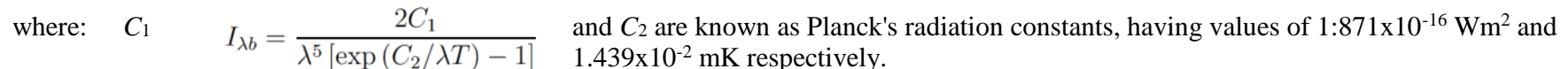

Real objects such as soot particles are not perfect emitters, and radiate less energy at each wavelength than the equivalent black body value. The spectral emissivity $\varepsilon_{\lambda}$ is defined as the ratio of the actual intensity emitted by a body at a given wavelength to the black body intensity. The emissivity of the flame has to be estimated and this can be done by the widely used empirical correlation established by Hottel and Broughton [11]

$$
\varepsilon_{\lambda}=1-\exp \left(-K L / \lambda^{\alpha}\right)
$$

where: $K$ is the absorption coefficient per unit flame thickness, and $L$ is the geometric flame thickness along the optical path length of the detection system.

The value of $\alpha$ depends on the physical and optical properties of the soot in the flame and, at visible wavelengths, was found by Hottel and Broughton [11] to have a value of 1.39 .

In conventional two-colour pyrometry the light sensing device is calibrated against a black body to determine the apparent temperatures at two specific wavelengths. The flame temperature can be estimated by assuming it to be a black body and combining equations 1 and 2 and solving for the ratio of the light intensities at two wavelengths. If the absolute intensities are known then an estimate of the $K L$ factor is also possible, but obtaining an absolute calibration of the light-sensing system is challenging.

In contrast, the colour-ratio pyrometry (CRP) technique [12] uses the ratios of the intensity of three colours to give two independent measures for determining the temperature and $K L$. The conventional approach normally uses narrow band filters and two light sensors/cameras with beam splitting optics, but it is also possible to use a single digital colour camera to avoid complexities such as the optical setup and image registration. The complication here is that a calibration is needed of the camera-specific colour filter array (Figure 6) and access to the raw data (not the reconstructed red, green and blue values for each pixel) [13]. The drawback with using the broadband filters in the colour filter array is that chemiluminescence will be a noise factor, but spectroscopic measurements in an ethylene diffusion flame show that these are of a much lower intensity than the thermal radiation so can be overlooked compared with other sources of error (such as uncertainty in the validity of equation 2) [14]. In order to make best use of the dynamic range of the camera a blue filter (Figure 6) can be used, since otherwise the blue pixel values can be significantly lower than the red pixel values. As cameras become more efficient the reduction in light reaching the red and blue pixels is not important. As the dynamic range of cameras increases the loss of information by taking ratios becomes less significant while the difficulty of making absolute measurements does not reduce. Nonetheless, if absolute measurements are feasible and can be combined with tomographic reconstruction, then limitations associated with the line of sight integration can be avoided and more complex models for the emissivity can be used, and it is possible to obtain information on temperature, soot volume fraction and particle size [14].

Page 4 of 12 

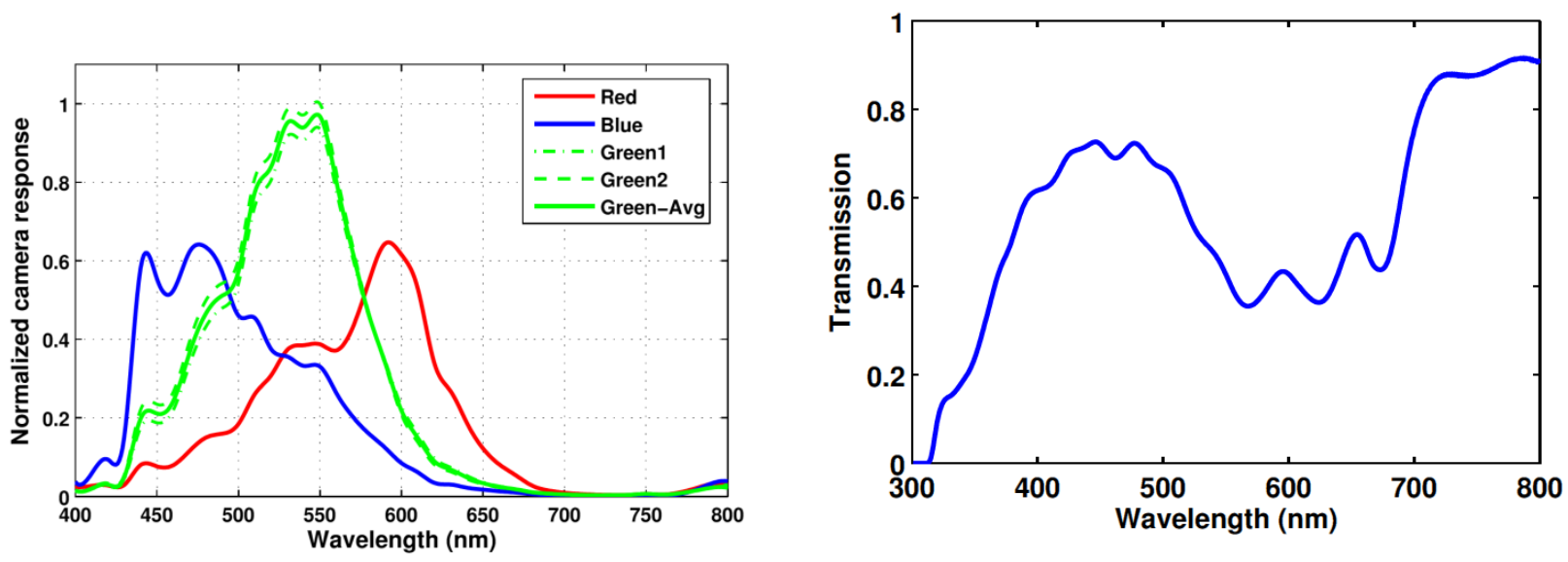

Figure 6 Calibration of the colour filter array (upper) and a blue filter (lower) that is used to increase the signal of the blue pixels relative to the green and red pixels.

The method for implementing three colour pyrometry is as follows:

a) For each combination of temperature and $K L$ that is of interest, use Equations 1 and 2 to compute the spectral intensity as a function of wavelength $I(T, K L)$,

b) At each wavelength, 'multiply' the spectral intensity, $I(T, K L)$, with the transmission of the filters for the red, green and blue pixels and integrate the result to obtain the camera response, $R(T, K L), G(T, K L)$ and $B(T, K L)$,

c) At each combination of temperature and $K L$ prepare look-up tables of the red/green and red/blue pixel ratios,

d) For each image, use interpolation from neighbouring pixels, so that for each pixel there is a red, green and blue value.

e) For each pixel in each image, evaluate the red/green and red/blue values, and use the look-up tables to find the best match for temperature and $K L$.

It is also necessary to check for saturated pixels (since this would invalidate the analysis), and to set a lower limit on pixel values since the calculations become ill-conditioned when a ratio is computed between two small numbers. Sensitivity studies are also needed, and these are likely to show that uncertainty on pixel values has a much greater adverse effect on the $K L$ value than the temperature. The parameter ' $K L$ ' is an integration of the soot loading across the path length, so integrating the $K L$ values across an image gives an indication of the total amount of soot that is present.

Figure 7 illustrates the use of full bore imaging combined with colour ratio pyrometry, and Figure 8 makes use of averaged and integrated data from these images. 

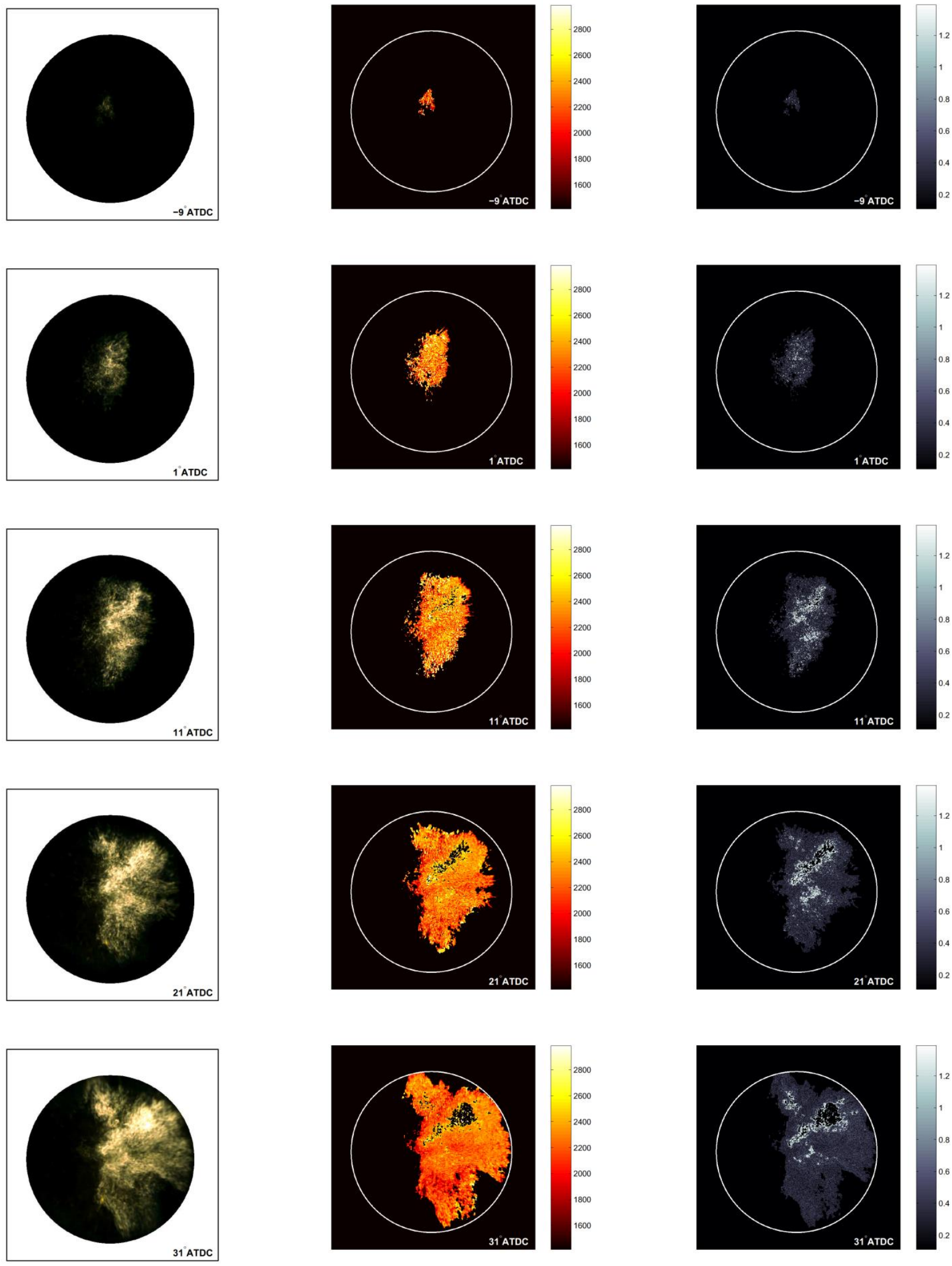

Figure 7 Example results of colour ratio pyrometry from tests with toluene and a lambda of 0.9 corrected flame image (left), temperature (middle) and $K L$ distribution (right).

Page 6 of 12 

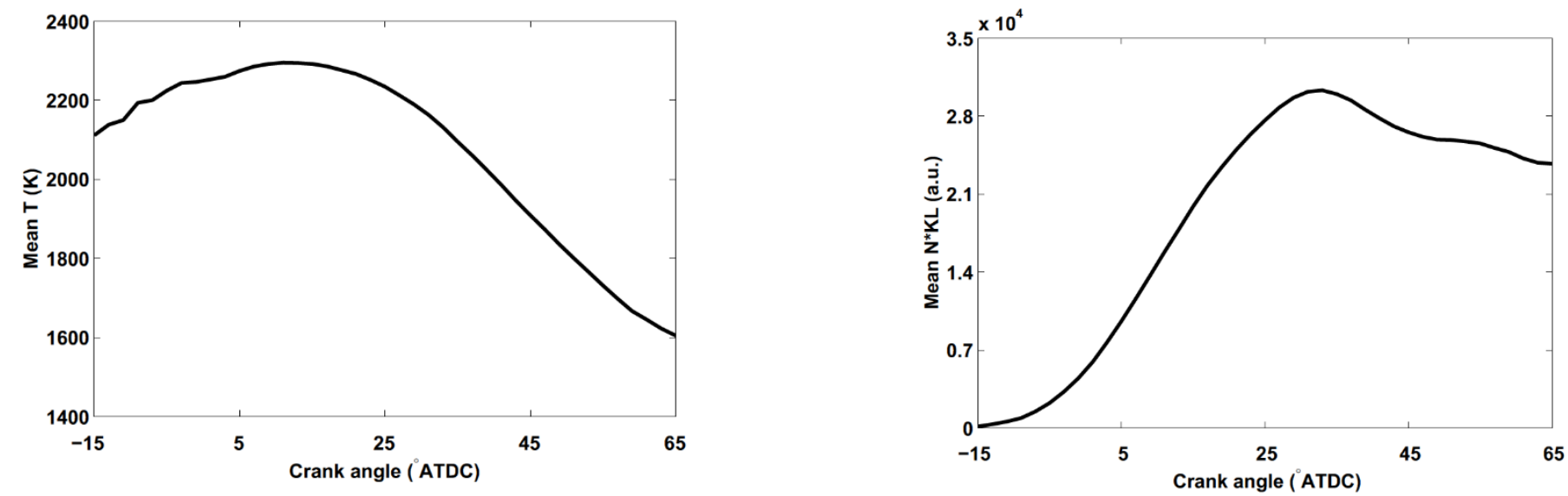

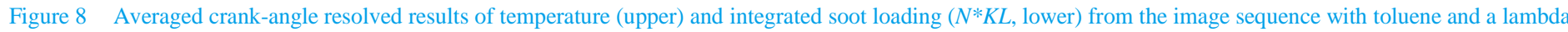
of 0.9 in Figure 7.

\section{Laser Based Techniques}

Laser based measurements for flow use well established techniques such as Laser Doppler Anemometry/Velocimetry (LDA/LDV) [15] for point measurements with a wide dynamic range and Particle Image Velocimetry (PIV) [16] that can provide 2D and indeed 3D measurements but with a lower dynamic range. Phase Doppler Anemometry (PDA) [17] allows particle sizes to be found as well as their velocity and it can be looked upon as an extension of LDA. Eckbreth [18] provides the details of several laser based techniques for combustion temperature and species measurements, such as Coherent Anti-Stokes Raman Spectroscopy (CARS), Spontaneous Raman and Rayleigh scattering, and Laser-Induced Fluorescence (LIF), with an emphasis on their use in combustion applications. As techniques for temperature and species measurements are less widely used they are to be discussed here.

\section{Temperature Measurements}

Although Rayleigh scattering can be used for planar temperature measurements the signal is relatively weak and particles must not be present in the flow, so other techniques are preferred. The first technique to gain prominence was Coherent Anti-Stokes Raman Spectroscopy (CARS), and this provides a point measurement. When a gas such as nitrogen is excited at appropriate wavelengths there is a spectroscopic emission at a characteristic wavelength with an intensity that depends on its temperature. By exciting the nitrogen at a number of different wavelengths the spectroscopic emission can be matched to library data. The early measurements used a dye laser that had to be scanned across a range of wavelengths, so this was only suited where the temperature field did not vary. Modern dual-broadband rotational CARS applied to an IC engine typically achieves $\pm 5 \%$ precision on single shot temperature [19]. Significantly improved precision could be achieved by employing a modeless broadband dye laser [20] which eliminates laser cavity mode noise and can achieve $\pm 1 \%$ under favourable lab conditions.

A more recent technique for gas-phase thermometry is Laser Induced Grating Spectroscopy (LIGS), and it can deliver an order of magnitude improvement with single shot temperature precision in the range $0.1-1.0 \%$ [21]. Two, short duration ( 1 - $10 \mathrm{~ns})$ pulses of laser light, of wavelength $\lambda$, intersect at a small angle $\theta$ to establish an interference pattern of bright and dark fringes. The separation of adjacent bright and dark

regions, or fringe width, $\Lambda$ is given by:

$$
\Lambda=\frac{\lambda}{2 \sin (\theta / 2)} \text {. }
$$

$$
\text { (3 }
$$

The optical arrangement is shown in Figure 9.

Figure 9 Experimental configuration for LIGS thermometry. (The optical access windows are not shown to scale.)

Energy absorbed by molecules in the bright fringes is transferred to the bulk gas and causes a small density perturbation. In the current example, toluene is used as a tracer since it will absorb light with a wavelength of $266 \mathrm{~nm}$ (the $4^{\text {th }}$ harmonic from the YAG laser). The resulting change in refractive index in the interference pattern is the laser induced grating. The grating has two components. Firstly, there is a stationary "temperature grating" and secondly, a standing wave "acoustic grating" arising from two counterpropagating sound waves induced by the sudden density perturbation. As

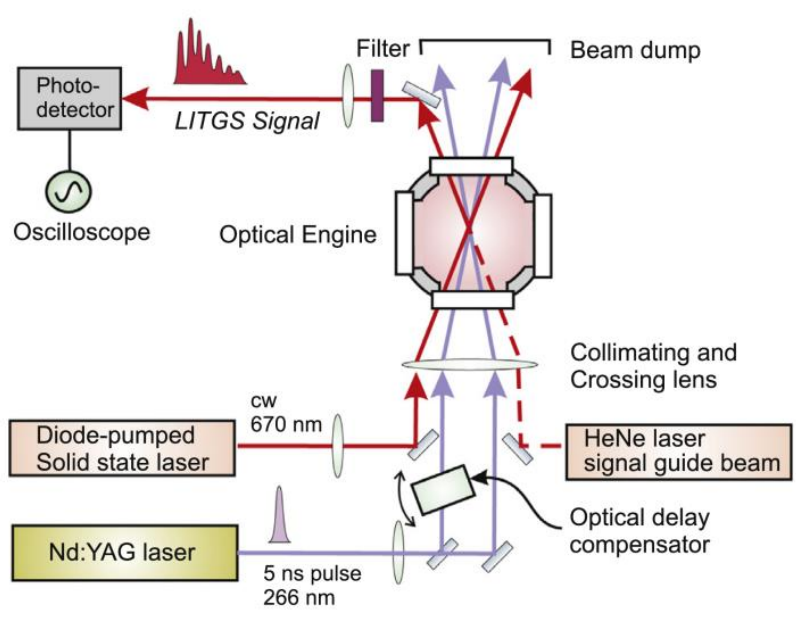

Page 7 of 12 
the acoustic oscillations periodically interfere constructively and destructively with the stationary grating the scattering efficiency of the grating is modulated in time at a frequency, $f_{\text {osc }}$, given by,

$$
f_{\text {osc }}=c_{s} / \Lambda
$$

where $c_{\mathrm{s}}$ is the speed of sound.

This oscillation frequency is measured by scattering a probe laser beam off the grating and measuring the temporal oscillations in scattered intensity. This probe laser can be of any convenient wavelength $\lambda_{\mathrm{P}}$ provided it is incident at the, so-called, Bragg angle, $\theta_{\mathrm{B}}$, given by:

$$
\theta_{B}=\sin ^{-1}\left(\lambda_{P} / 2 \Lambda\right)
$$

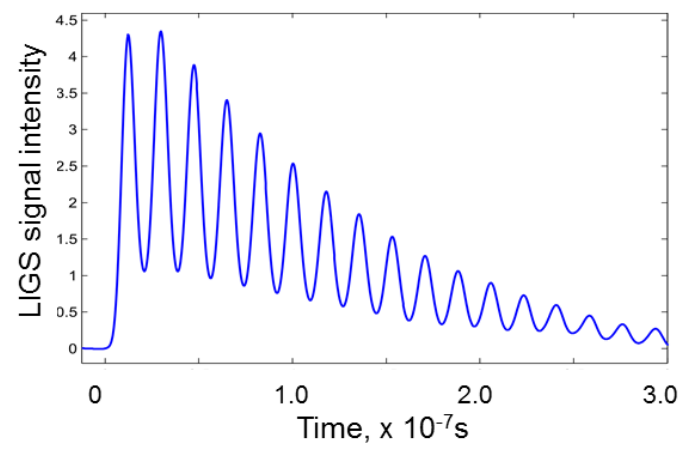

The LIGS signal is the scattered light and it is a coherent laser-like beam, with a temporal variation at $f_{\text {osc }}$, as illustrated schematically in Figure 10. The decay rate decreases with increasing pressure leading to longer signals and increased precision in the determination of the oscillation frequency at higher pressures. The $\mathrm{HeNe}$ laser is only used to align the location of the detector for the LIGS signal. It is also worth noting that the detector can be placed remotely from the grating since there is no loss of signal intensity while any scattered light is reduced in accordance with the inverse square law.

Figure 10 Temporal form of a typical LIGS signal (excitation at time zero).

The temperature can be found because of the relationship between the speed of sound and temperature in a gas of known composition:

$c_{S}=\left(\frac{\gamma R_{o} T}{M}\right)^{1 / 2}$

Where $\gamma$ is the ratio of specific heats at constant volume and pressure, $M$ is the mean molar mass and $R_{\mathrm{o}}$ is the molar gas constant. Hence, from the measured oscillation frequency, $f_{\text {osc}}$, the temperature is derived from:

$T=\frac{M}{\gamma} \frac{\Lambda^{2}}{R_{0}} f_{o s c}^{2}$

Since the temperature is derived from a measurement of frequency rather than intensity, the precision is no longer limited by the signal intensity or noise.

Errors due to variation in composition can be quantified if the range of variations from an assumed composition of air, EGR and fuel, can be estimated with reasonable confidence. It is found that for in-cylinder gases the main compositional uncertainties arise from variation in the fuel/air ratio and its homogeneity; the amount of EGR is much less important. Fortunately the variation of $\gamma R$ with temperature is found to be insignificant over the temperature range encountered in these experiments. More details of the calculations of compositional uncertainties is presented in a previous publication [21], which also reports LIGS measurements showing the influence of alcohol content level on the mixture temperature during compression in a firing engine.

\section{Planar Laser Induced Fluorescence (PLIF)}

Planar Laser Induced Fluorescence (PLIF) can be used for a number of different applications, including: location of the flame front, air fuel ratio measurements and temperature measurement. Light from a laser can be readily expanded into a sheet by the use of cylindrical lenses, and this provides a planar illumination source. Often UV light is used, since many materials fluoresce when subject to UV light and that is indeed also a source of noise when there is stray fluorescence. The narrow band filter (typically $10 \mathrm{~nm}$ bandwidth) used to block the excitation laser light can help eliminate stray fluorescence. As the fluorescence intensity is weak and it is further attenuated by the filter, then imaging requires an intensified camera, such as an ICCD (Intensified Charge Coupled Device). 
If PLIF is being used to find the location of a flame front, then one approach is to detect $\mathrm{OH}$ since this only occurs in the burned gas. OH can be detected by using the second harmonic $(283 \mathrm{~nm})$ from a dye laser $(566 \mathrm{~nm})$ that has been pumped by the second harmonic of a YAG laser (532nm); the $\mathrm{OH}$ fluorescence is then detected in the $300-330 \mathrm{~nm}$ range. This avoids the ambiguities associated with line-of-sight integration from natural light photography (chemiluminescence), but will be likely to have a lower frame rate and resolution. The concentration of $\mathrm{OH}$ increases very rapidly with temperature, but trying to extract the temperature is confounded by the fluorescence spectra being a function of temperature, and the immense difficulty in trying to calibrate the PLIF image, since this will require:

a) knowledge of the intensity of the laser sheet

b) knowledge of the fluorescence efficiency at the detection wavelength for the particular excitation wavelength and local bulk-gas collision partners

PLIF has been widely used for recording 2D measurements of in-cylinder fuel distributions, as there is potential for quantitative measurement of the air fuel ratio or equivalence ratio. A non-fluorescing fuel has to be doped with another compound (the tracer) that does fluoresce. The photophysics relevant to quantitative planar laser-induced fluorescence, Q-PLIF, has been treated in a detailed review by Schulz and Sick [22]. The intensity of the fluorescence (that has been excited by a suitable laser pulse) is confounded by a number of factors, not least the dependence of the fluorescence yield on temperature, pressure and local composition.

Quantitative interpretation of PLIF images requires the correct treatment of many parameters; consider the form of the equation for predicting the LIF signal, $S_{\mathrm{f}}$

$$
S_{\mathrm{f}} \propto n \times I \times \sigma(T) \times \varepsilon_{\mathrm{fl}}(T, p, \text { composition) }
$$

Where: $n$ is the number density of the absorbing molecules, $I$ is the laser intensity (which might not be uniform across the laser sheet), $\sigma(T)$ is the temperature dependent absorption cross-section and $\varepsilon_{\mathrm{fl}}(T, p$, composition) is the fluorescence quantum efficiency which depends on temperature, $T$, pressure $p$ and gas composition.

This equation is only valid in the linear regime, when the fluorescence transition is not saturated. In other words, the laser intensity $I$ must not be too high. Furthermore, the tracer concentration must be sufficiently low for the mixture through which the laser beam propagates to be optically thin that is, minimal absorption of the laser beam should occur.

The fuel and tracer also need to co-evaporate (or otherwise the concentration of the tracer will be known and not the fuel). Table 2 shows a fuel with three different tracers that can co-evaporate with the light medium and heavy components in a model fuel that represents the boiling point range of a gasoline [23].

Table 2 Fuel composition and corresponding tracer, doping fractions and spectral properties. (The spectral properties are dependent on temperature and composition so the figures here are only a guide).

\begin{tabular}{|c|c|c|c|c|}
\hline \multicolumn{2}{|c|}{$\begin{array}{l}\text { Component } \\
\text { Vol fraction } \\
(\%)\end{array}$} & $\begin{array}{l}\text { Tracer } \\
\qquad \text { Vol fraction } \\
(\%)\end{array}$ & \multirow{2}{*}{$\begin{array}{c}\begin{array}{c}\text { Excitation } \\
\text { wavelength } \\
(\mathrm{nm})\end{array} \\
277\end{array}$} & \multirow{2}{*}{$\begin{array}{c}\begin{array}{c}\text { Fluorescence } \\
\text { wavelength } \\
(\mathrm{nm})\end{array} \\
350-550\end{array}$} \\
\hline Butanes & 0.144 & Acetone 5 & & \\
\hline Isopentanes & 0.166 & & & \\
\hline Iso-octane & 0.454 & Toluene 2 & 248 & $270-330$ \\
\hline n-Octane & 0.112 & & & \\
\hline Iso-dodecane & 0.022 & 1,2,4 Tri- 2 & 248 & $270-350$ \\
\hline n-Decanes & 0.101 & methyl benzen & TMB) & \\
\hline
\end{tabular}

It is also necessary to have a calibration, and this is best achieved in situ with a homogeneous mixture. If the engine is motored with a recirculating loop, in which the mixture can be heated and pressurised, then the compression process enables the PLIF signal to be calibrated over a wide range of temperatures and pressures. The mixture composition can be determined by using a FID to measure the hydrocarbon concentration.

In the firing engine exhaust gas residuals in the pre-combustion mixture may affect the efficiency of the PLIF, and invalidate the calibration made in their absence. To account for these effects an additional calibration scheme is to take PLIF images of homogeneous charge distributions produced by plenum injection in firing cycles at the start and end of an experimental run. These "book end" calibration images are thus obtained "on the fly". As a result, any changes in transmission of the windows are automatically accounted for and exhaust gas residuals will also be present in the calibration images at the same levels as when direct injection is used. Because the exciting laser has a spatially non-uniform beam profile, then this needs to be monitored so that the resulting images from non-uniform excitation can be normalised. If all of these precautions are taken, then Quantitative PLIF (QPLIF) can be achieved, with some example images shown in Figure 11. A comparatively late injection timing has been used as this leads to more stratification and structure in the images. 

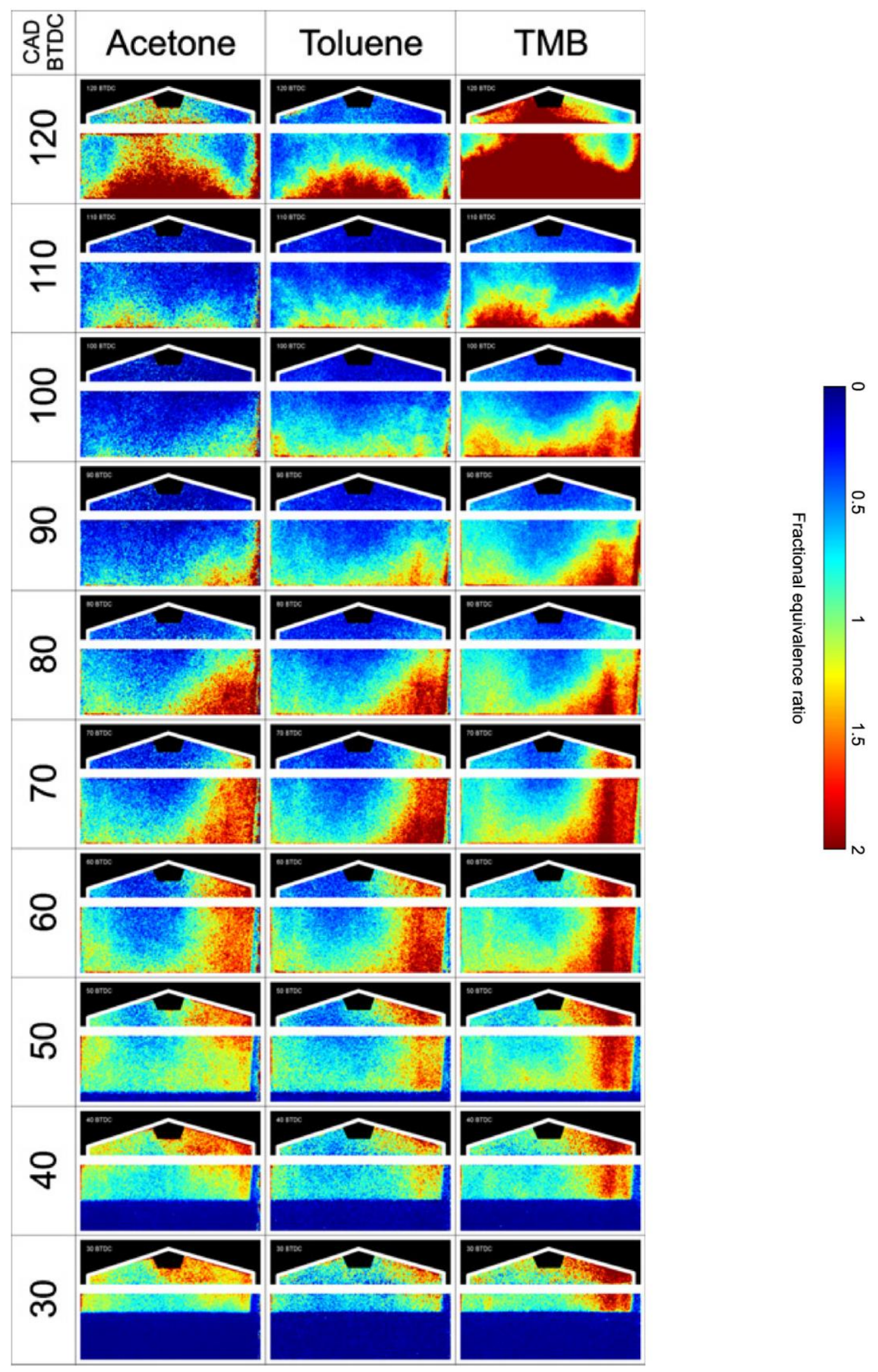

Figure 11 QPLIF images formed by each of three tracers: acetone, toluene and trimethylbenzene (TMB). Each image is a 32-frame average with injection timing at $140^{\circ} \mathrm{ca}$ bTDC (the density of the plume of injected fuel precludes quantitative analysis at or before $120^{\circ} \mathrm{ca}$ ). The top of the piston is seen intruding into the images from $50^{\circ} \mathrm{ca}$ onwards. The images have been corrected for window transmission factors and scaled using the "book end" calibration images to give $2 \mathrm{D}$ distributions of the fractional equivalence ratio for light, medium and heavy fuel components.

Page 10 of 12 
The temperature dependence of the fluorescence means that there is potential for using PLIF to deduce temperature if there are two independent measurements of the fluorescence. This leads to various possibilities incuding: i) excite the tracer at two different wavelengths and record the fluorescence from each excitation, or ii) excite the tracer at a single wavelength but record the fluorescence at two different wavelengths. Exciting the tracer at a single wavelength means that the laser intensity is automatically the same at every location within the laser sheet so all that matters is the relative fluorescence intensity at the two different wavelengths. Figure 12 shows how the fluorescence intensity from toluene varies with temperature with excitation at $266 \mathrm{~nm}$. Also identified in Figure 12 are two wavelengths (centred on $270 \mathrm{~nm}$ and $300 \mathrm{~nm}$ ) which have an opposite response to increasing temperature, so that if the ratio of the two signals is taken they will give an indication of temperature that is independent of the tracer concentration and excitation intensity (so long as there is no saturation). The necessary optics for this T-PLIF technique are shown in Figure 13 , and comprise a dichroic mirror that acts as a beam splitter with additional narrow band filters ('red' and 'blue') and ICCD cameras.

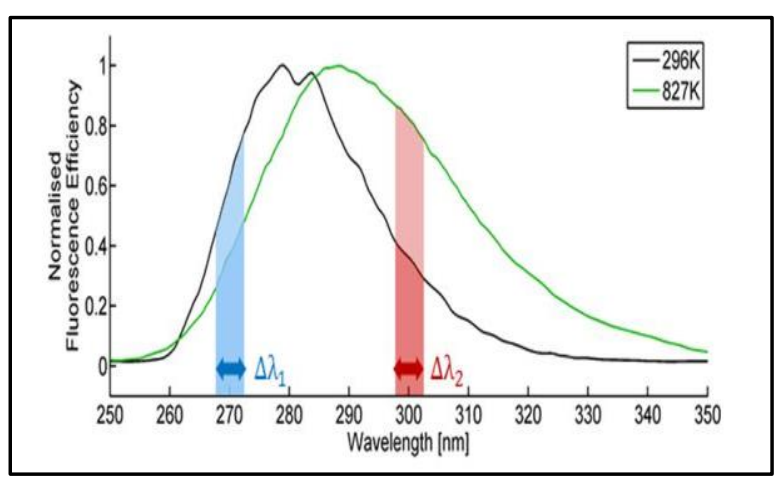

Figure 12 The dependence of fluorescence intensity from toluene with temperature when excited at $266 \mathrm{~nm}$ [24]

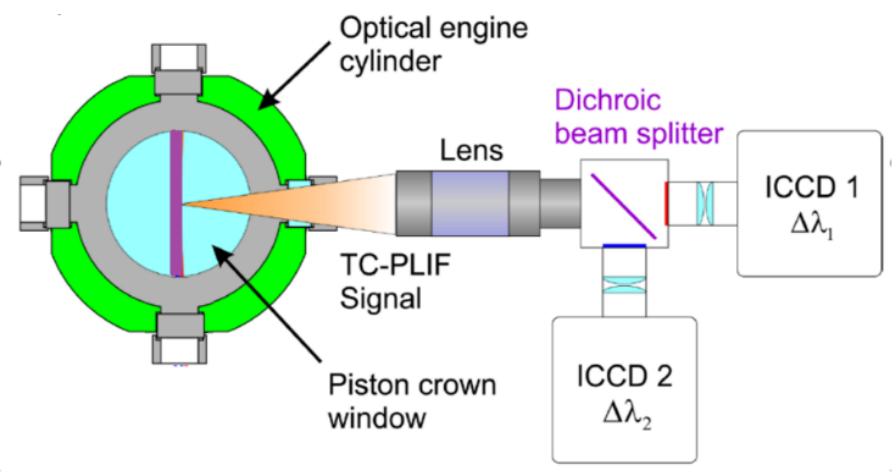

Figure 13 An optical system to image the fluorescence from the tracer in the laser sheet, with a dichroic mirror beam splitter and additional narrow band filters ('red' and 'blue') for each of the ICCD cameras.

The ratio of the 'red' and 'blue' ICCD camera images depends on temperature, but the dependency will be more complex than the calibration needed for QPLIF. However, in situ calibration of 2D T-PLIF measurements can be achieved by using a second independent technique (such as a LIGS point measurement); for more comprehensive details there is a related paper [25].

\section{Conclusions}

Optical techniques can provide data that is useful for validation of modelling and elucidating the processes of mixture preparation and combustion. The results presented here are for illustration of the techniques, and more comprehensive measurements and their use can be found in the papers that have been referenced as each technique is described. There are of course differences in the performance of 'thermodynamic' engines (that is, conventional metal engines) and optical engines, but modelling can be used to bridge this gap. The simplest techniques rely on high speed photography, either with natural light or with an illumination source. There is of course a limitation caused by the line of sight integration, but none the less it is possible to obtain information that is semi-quantitative, for example:

- use can be made of the scattered light in Mie scattering to give an indication of the quantity of liquid fuel that is present,

- $\quad$ flame front tracking can be used to estimate enflamed area and flame front velocity,

- $\quad$ soot pyrometry can be used to estimate temperature fields and the quantity of soot that is present.

If there is a single jet of fuel, then measuring the spray angle and penetration should be unambiguous, but two simultaneous images will be needed unless the orientation of the spray is known.

Of the laser techniques, Planar Laser Induced Fluorescence (PLIF) is comparatively easy to implement as a sheet of UV laser light will make almost anything fluoresce, and that is also its disadvantage - it is very difficult to make PLIF a quantitative technique since the fluorescence detected by an image-intensified camera depends on numerous factors, such as: the intensity of the excitation, the fluorescence yield and the sensitivity of the detection system. In contrast, non-linear techniques such as Laser Induced Grating Spectroscopy (LIGS) can be more difficult to generate and collect a signal, but once the signal is collected, then its interpretation is less ambiguous.

As lasers become cheaper, more compact, and with higher repetition rates their use can become more widespread. At the same time high speed cameras are having increased sensitivity, greater resolution and a larger dynamic range. These two trends combine to mean that optical diagnostic will be of ever increasing importance in combustion research.

\section{References}

1 Bowditch F.W., "A New Tool for Combustion Engine Research-A Quartz Piston Engine”, SAE Technical Paper $610002,1961$.

2 Zhao, H. Laser diagnostics and optical measurement techniques in internal combustion engines. SAE 2012.

Page 11 of 12 
Sandford, M., Page, G., and Crawford, P. “The all new AJV8”. SAE Technical Paper 2009-01-1060, 2009.

4 Murad, S. H. M, Camm, J., Davy, M., Stone, R., et al. "Spray Behaviour and Particulate Matter Emissions with M15 Methanol/Gasoline Blends in a GDI Engine”. SAE Technical Paper 2016-01-0991, 2016.

5 Chen, L., Stone, R., and Richardson, D. "A study of mixture preparation and PM emissions using a direct injection engine fuelled with stoichiometric gasoline/ethanol blends." Fuel 96: 120-130, 2012.

6 Le Gal, P., Farrugia, N., and Greenhalgh, D. A., "Laser sheet dropsizing of dense sprays.” Optics \& Laser Technology, 31(1), 75-83, 1999.

7 Twiney, B., Stone, R., Chen, X., and Edmunds, G., "Investigation of Combustion Robustness in Catalyst Heating Operation on a Spray Guided DISI Engine, Part II-Measurements of Spray Development, Combustion Imaging and Emissions." SAE Int. J. Engines 3,: 426-439. SAE 201001-0603, 2010.

8 Fansler, T. D., Stojkovic, B., Drake, M. C., and Rosalik, M. E. "Local fuel concentration measurements in internal combustion engines using spark-emission spectroscopy." Applied Physics B 75, no. 4-5: 577-590, 2002.

9 Ma, H., Marshall, S., Stevens, R., and Stone, R. "Full-bore crank-angle resolved imaging of combustion in a four-stroke gasoline direct injection engine." Proc. IMechE Part D: Journal of Automobile Engineering 221, no. 10: 1305-1320, 2007.

10 Perona, P., and Malik, J. "Scale-space and edge detection using anisotropic diffusion". IEEE Transactions on pattern analysis and machine intelligence, 12(7), 629-639, 1990.

11 Hottel, H.C., and Broughton, F.P., "Determination of true temperature and total radiation from luminous gas flames". Industrial \& Engineering Chemistry Analytical Edition, 4(2), pp.166-175, 1932.

12 Simonini, S., Elston, S. J., and Stone, C. R. "Soot temperature and concentration measurements from colour charge coupled device camera images using a three-colour method." Proc. IMechE Part C: Journal of Mechanical Engineering Science 215, no. 9: 1041-1052, 2001.

13 https://en.wikipedia.org/wiki/Bayer_filter accessed 19 January 2017

14 Zhao, H., Williams, B., and Stone, R. "Measurement of the spatially distributed temperature and soot loadings in a laminar diffusion flame using a Cone-Beam Tomography technique." J. Quantitative Spectroscopy and Radiative Transfer 133: 136-152, 2014.

15 https://en.wikipedia.org/wiki/Laser_Doppler_velocimetry accessed 19 January 2017.

16 https://en.wikipedia.org/wiki/Particle_image_velocimetry accessed 19 January 2017.

17 http://www.dantecdynamics.com/measurement-principles-of-pda accessed 19 January 2017.

18 Eckbreth, A. C., Laser Diagnostics for Combustion Temperature and Species, Gordon and Breach Science Publishers SA, Amsterdam, 1996, pp.209-451.

19 Brackmann, C., Bood, J., Afzelius, M., and Bengtsson, P-E., "Thermometry in internal combustion engines via dual-broadband rotational coherent anti-Stokes Raman spectroscopy” Meas. Sci. Technol. 15, 2004.

20 Snowdon, P., Skippon, S. M. and Ewart, P., "Improved precision of single-shot temperature measurements by use of a modeless laser," Appl. Optics, 30, 1008, 1991.

21 Williams, B, Edwards, M., Stone, R., Williams, J., and Ewart, P., "High precision in-cylinder gas thermometry using Laser Induced Gratings: Quantitative measurement of evaporative cooling with gasoline/alcohol blends in a GDI optical engine," Combustion and Flame 161, 270279, 2014

22 Schulz, C. and Sick, V., "Tracer-LIF diagnostics: quantitative measurement of fuel concentration, temperature and fuel/air ratio in practical combustion systems," Prog Energy and Combust Sci, 31(1), pp.75-121, 2005.

23 Williams, B., Ewart, P., Wang, X., Stone, R., et al., "Quantitative planar laser-induced fluorescence imaging of multi-component fuel/air mixing in a firing gasoline-direct-injection engine: effects of residual exhaust gas on quantitative PLIF," Combustion and Flame, 157(10), pp.1866-1878, 2010.

24 Faust, S., Tea, G., Dreier, T. and Schulz, C., "Temperature, pressure, and bath gas composition dependence of fluorescence spectra and fluorescence lifetimes of toluene and naphthalene." Applied Physics B, 110(1), pp.81-93, 2013.

25 Scott, B., Willman, C., Williams, B., Ewart, P., et al., "In-cylinder Temperature Measurements using Laser Induced Grating Spectroscopy and Two-Colour PLIF”, 17ICE-0047, SAE Naples, 2017.

\section{Contact Information}

Richard Stone

Department of Engineering Science

Parks Rd

OXFORD OX1 3PJ

+44(0)1865273139

richard.stone@eng.ox.ac.uk

\section{Acknowledgments}

Funding is gratefully acknowledged from EPSRC (partially funded from EP/I011331/1, EP/H049967/1, EP/G000565/1, GR/S67227/01, GR/S58829/01), Jaguar Land Rover, Shell Global Solutions and BP. 